
\title{
Resarach Suate \\ Observation of light induced dynamical band structure via multi-band high harmonic spectroscopy
}

Ayelet Uzan-Narovlansky ( $\square$ auzan@princeton.edu )

Princeton University

\section{Alvaro Jimenez-Galan}

Max-Born-Institute for Nonlinear Optics and Short Pulse Spectroscopy https://orcid.org/0000-0003-

2116-7187

\section{Gal Orenstein}

Weizmann Institute of Science

\section{Rui Silva}

Universidad Autónoma de Madrid

\section{Talya Arusi-Parpar}

Weizmann Institute of Science

\section{Sergei Shames}

Weizmann Institute of Science

\section{Barry Bruner}

Weizmann institute of science

\section{Binghai Yan}

Weizmann Institute of Science https://orcid.org/0000-0003-2164-5839

\section{Olga Smirnova}

Max Born Institute

Misha (Mikhail) Ivanov

Max-Born Institute https://orcid.org/0000-0002-8817-2469

\section{Nirit Dudovich}

Weizmann Institute of Science https://orcid.org/0000-0003-4996-4743

\section{Research Article}

Keywords: Intense light-matter interactions, all-optical spectroscopy, conduction bands

Posted Date: August 31st, 2021

DOI: https://doi.org/10.21203/rs.3.rs-803528/v1 
License: (c) (i) This work is licensed under a Creative Commons Attribution 4.0 International License. Read Full License

Version of Record: A version of this preprint was published at Nature Photonics on June $2 n d, 2022$. See the published version at https://doi.org/10.1038/s41566-022-01010-1. 


\title{
Observation of light induced dynamical band structure via multi-band high harmonic spectroscopy
}

\author{
Ayelet J. Uzan-Narovlansky ${ }^{1,2}$, Álvaro Jiménez-Galán ${ }^{3}$, Gal \\ Orenstein $^{4}$, Rui E.F. Silva ${ }^{5}$, Talya Arusi-Parpar ${ }^{1}$, Sergei Shames ${ }^{1}$, \\ Barry D. Bruner ${ }^{1}$, Binghai Yan $^{6}$, Olga Smirnova ${ }^{3,7}$, Misha \\ Ivanov $^{3,8,9}$ and Nirit Dudovich ${ }^{1}$ \\ ${ }^{1}$ Department of Complex Systems, Weizmann Institute of Science, 76100, Rehovot, Israel \\ ${ }^{2}$ Department of Physics, Princeton University, Princeton, NJ, USA \\ ${ }^{3}$ Max-Born-Institut, Max-Born Strasse 2A, D-12489 Berlin, Germany \\ ${ }^{4}$ SLAC National Accelerator Laboratory, Stanford University, Stanford, California 94305, USA \\ ${ }^{5}$ Department of Theoretical Condensed Matter Physics, Universidad Autónoma de Madrid, \\ E-28049 Madrid, Spain \\ ${ }^{6}$ Department of Condensed Matter, Weizmann Institute of Science, 76100, Rehovot, Israel \\ ${ }^{7}$ Technische Universität Berlin, Ernst-Ruska-Gebäude, Hardenbergstr. 36A, D-10623 Berlin, \\ Germany \\ ${ }^{8}$ Blackett Laboratory, Imperial College London, South Kensington Campus, SW7 2AZ London, \\ United Kingdom \\ ${ }^{9}$ Department of Physics, Humboldt University, Newtonstrasse 15, 12489 Berlin, Germany
}

August 3, 2021 
Intense light-matter interactions have revolutionized our ability to probe and manipulate quantum systems at sub-femtosecond time scales ${ }^{[1]}$, opening routes to all-optical control of electronic currents in solids at petahertz rates, the time-scale of a single optical cycle ${ }^{[2,3,4,5,6,7]}$. Such control typically requires electric field amplitudes $\sim V / \AA$, when the voltage drop across a lattice site becomes comparable to the characteristic band gap energies. In this regime, intense light-matter interaction induces significant modifications of electronic and optical properties ${ }^{[8,9,10]}$ and is certain to dramatically modify the band structure of the lightdressed crystal. Yet, identifying and characterizing such modifications remains an outstanding problem. As the oscillating electric field changes from $\sim V / \AA$ to zero within a quarter-cycle of the driving field, does the band-structure follow, and how can it be defined? Here we address this fundamental question, proposing all-optical spectroscopy of strongly driven crystals, and probe laser-induced closing of the bandgap between adjacent conduction bands. Our work reveals the link between extreme nonlinear light matter interactions in strongly driven crystals to the sub-cycle modifications in their effective band structure.

\section{Main}

The ability of near-resonant light fields to modify the energetics and the dynamics of an atom (or a molecule), is central to many phenomena such as laser cooling, trapping, and quantum optics of atoms in a cavity ${ }^{[1,12]}$, electromagnetically induced transparency ${ }^{[13]}$, slow light ${ }^{[14,15]}$, or extremely efficient generation of hyperRaman lines ${ }^{[16]}$. The combined states of matter and near-resonant light are generally viewed over many laser cycles, using the Floquet formalism which takes advantage of the periodicity of light oscillations. In solids, cycle-averaged modification of the hopping rates between the neighboring sites leads to such spectacular manifestations as coherent destruction of tunneling ${ }^{[17]}$ and modified local interaction potentials for the electron motion in the conduction bands ${ }^{[10]}$.

The situation changes in light fields with frequencies well below resonance but with intensities sufficiently high to induce electron-volts-scale voltages across a lattice site. This regime is attractive technologically, as it enables control of 
electron dynamics on the sub-cycle time-scale, i.e. potentially at peta-hertz rates, in solids. As the oscillating electric field of the lightwave changes from zero to its maximum value within a quarter-cycle, rapidly changing voltages can lead to subcycle modifications of the macroscopic properties, such as the transmittance ${ }^{[4,6]}$ or conductance ${ }^{[7]}$. At the same time, with the instantaneous voltages changing on a sub-cycle scale, the cycle-averaged frequency-domain Floquet perspective is hardly satisfying.

Here we describe how the effective band structure can be introduced in such interaction regime within a time-domain perspective. We experimentally demonstrate all-optical spectroscopy of a strongly driven crystal, revealing an anomalous spectral intensity response. Our theoretical study links these observations to laser-induced closing of the gap between adjacent conduction bands (fig. 1a). Our experimental approach is based on high harmonic generation (HHG) spectroscopy, which maps the instantaneous coherent properties of matter onto the HHG spectrum. Solid state HHG ${ }^{[18]}$ opened the door to all-optical spectroscopy of electronic structure and dynamics in crystals ${ }^{[18,19,20,21,22,23,24]}$, many-body ${ }^{[25]}$ or topological phenomena ${ }^{[8,9,26,27]}$, including all-optical spectroscopy of $2 \mathrm{D}$ materials ${ }^{[27,28]}$.

High harmonic generation in solids involves optical tunneling ${ }^{[29]}$ across the gap between the valence and the conduction band. This transition initiates intraband harmonics associated both with the tunneling current across the band gap ${ }^{[30]}$ and the Bloch oscillations that follow ${ }^{[10,18]}$, while the electron-hole recombination leads to the higher-order harmonic emission ${ }^{[31]}$. At high light intensities and/or low frequencies, one enters a new dynamical regime. In many systems, as the electronhole wavepacket approaches the edges of the Brillouin zone, Landau-Dykhne-type transitions ${ }^{[32]}$ can promote electrons to higher conduction bands, as reflected in the harmonic spectra ${ }^{[21,33]}$ (fig. 1a). However, can the imprint of these transitions on high harmonic emission be used to observe light-induced modifications of the bands?

One can show that light-induced modifications of the bands are directly linked to the sub-cycle Landau-Dykhne-type transitions between them. Details of the theoretical analysis, which extends the Floquet-type analysis into the sub-cycle domain, are presented in the Methods section; here we summarize the main results. In the low-frequency limit, one begins with the adiabatic approximation, which treats the phase $\omega t$ of the electric field oscillations as a parameter, and finds the 
adiabatic band structure $\epsilon(k, \omega t)$ in the presence of a quasistatic electric field. When a non-adiabatic Landau-Dykhne type transition between a pair of bands occurs, typically as the electron is laser-driven across the minimal band-gap, it modifies the gap as follows (see Methods section for derivation):

$$
\Delta \epsilon_{\mathrm{eff}}(t) \simeq \Delta \epsilon_{\mathrm{ad}}(t) \sqrt{\frac{1-w_{\mathrm{LD}}(t)}{1+w_{\mathrm{LD}}(t)}}
$$

where $\Delta \epsilon_{\text {eff }}$ is the effective bandgap, $\Delta \epsilon_{\text {ad }}$ is the bandgap in the adiabatic approximation, and $w_{\mathrm{LD}}$ is the probability of the sub-cycle Landau-Dykhne type transition. The result is intuitive: the effective band gap is closed by the laser field when the sub-cycle transition approaches unity, $w_{\mathrm{LD}}(t) \simeq 1$. Given the exponential sensitivity of $w_{\mathrm{LD}}(t)$ to laser field strength (see Methods), the effective bandgap closes soon after the sub-cycle excitation probability becomes appreciable.

To investigate this phenomenon experimentally, we use the two-color HHG spectroscopy ${ }^{[34,35]}$ (fig. 1b), augmenting strong fundamental driver with a weak second harmonic (SH) field, while controlling their subcycle delay $\tau$. Electron-hole trajectories responsible for different harmonics ${ }^{[36]}$ are perturbed by the weak field: each trajectory acquires an additional complex phase $\sigma(\tau)$, which is accumulated along the entire trajectory, serving as a sensitive label of its temporal properties. If the fundamental field generates only odd harmonics, the SH field breaks the symmetry of the interaction, as $\sigma(\tau)$ changes sign between the two consecutive half cycles (see SI). This phase is mapped into the harmonic intensity as:

$$
\begin{array}{r}
I_{\text {odd }}(\sigma(\tau)) \propto\left(e^{i \sigma}+e^{-i \sigma}\right) \\
I_{\text {even }}(\sigma(\tau)) \propto\left(e^{i \sigma}-e^{-i \sigma}\right)
\end{array}
$$

Scanning $\tau$ modifies $\sigma(\tau)$ in a periodic manner, modulating the harmonic spectrum. The modulation phase and contrast encode the dynamical properties of electron trajectories associated with each harmonic order and allow their reconstruction with attosecond precision. In gas phase, the two-color HHG spectroscopy revealed the underlying dynamics of field-induced tunneling ${ }^{[35]}$, multielectron rearrangement and hole dynamics ${ }^{[37]}$. In solids, the two-color experiments resolved the interband contribution to high-harmonic emission ${ }^{[36]}$ and provided all-optical reconstruction of the field-free band structure upon exciting a single conduction 
band $^{[19]}$. Recently this approach has been applied to resolve the nonlinear interaction associated with solid state HHG in transmission and reflection configurations ${ }^{[38]}$. In this paper we use this scheme to study the underlying dynamics of driven multiband currents, probing the origin field dressed band structure.

Experiments were performed on $\mathrm{MgO}^{[39]}$, using $\lambda=1.3 \mu \mathrm{m}$ laser field at intensities $\sim 10^{13} \frac{\mathrm{W}}{\mathrm{cm}^{2}}$ and a weak SH field, polarized parallel to the fundamental field. We have measured the HHG modulations with the two-color delay and extracted the oscillation phase $\Phi_{N}$ associated with each harmonic order $N$ (see SI). Figure 2 presents the oscillation phase $\Phi_{N}$ as a function of harmonic order for orientation angles of 0 degrees $(\Gamma X)$ and 45 degrees $(\Gamma K)$, with respect the fundamental field's polarization. For harmonics $N=11-15(10.5-14.5 \mathrm{eV})$, which are associated with the electron-hole recombination from the first conduction band, we measure a gradual slope of $\Phi_{N}$ with $N$. This observation is in close agreement with our semiclassical calculations, which use a trajectory-based approach ${ }^{[36]}$ and associate each harmonic with a dominant trajectory within a single conduction band (see SI). The slope reflects the evolution of the trajectory length with the harmonic order ${ }^{[36]}$. As we approach the edge of the Brillouin zone, represented by harmonic $17(16 \mathrm{eV})$, this simple description fails. A clear phase jump appears in the measurements, associated with the edge of the zone and the changing bands curvature. At this point the mapping between momentum and energy becomes singular, leading to the appearance of enhanced spectral feature, previously identified as a spectral caustic ${ }^{[21]}$. The two-color phase measurement resolves this transition and the appearance of caustics, associated with the interference of multiple electron-hole trajectories. Thus, the phase measurement serves as sensitive probe of the band gap and allows its accurate identification.

Beyond the cut-off energy of the first conduction band, the electron dynamics involve multiple bands ${ }^{[20,33]}$. The harmonic emission is dictated by electron currents originating from higher conduction bands, determined by their structure and coupling. Importantly, these parameters depend on the crystal orientation, with each orientation offering a new one-dimensional slice of the band structure ${ }^{[24,39]}$. We can thus track the dependence of electron dynamics on the band structure and laser-induced couplings by resolving the oscillation phase of each harmonic as a function of the crystal orientation.

Figure 3 a presents the HHG oscillation phase, for various crystal orientations. 
The oscillation phases of the lower harmonics (H11,H13,H15) emitted far from the edge of the Brillouin zone remain unchanged with the crystal orientation. Indeed, the bottom of the first conduction band, as well as its distance from the valence band, $\epsilon_{c 1, v}=\epsilon_{c 1}-\epsilon_{v}$, is approximately isotropic (fig. 3c).

For higher harmonics $(N=19-29)$, a strong variation of the oscillation phase with crystal orientation is observed (fig. 3a). Figure 3b presents the oscillation phase as a function of the crystal's orientation for H21. The phase shows a sharp increase by more than $\pi$ as the orientation angle changes towards 45 degrees. The origin of this sharp variation can be understood by looking at the angular dependence of the bands. Figure 3c shows the energy difference between the valence, the first, and the second conduction bands $\left(\epsilon_{c 1, v}, \epsilon_{c 2, v}\right)$ for different crystal orientations, together with the energy contours for harmonic 21 . The crystal momentum at the emission point associated with $\mathrm{H} 21$ changes quickly with crystal's orientation, as illustrated in fig. 3d. The strong orientation-dependent modifications of the band gap, $\epsilon_{c 2, v}$, lead to significant angular changes of the corresponding electron trajectories, as captured by the phase measurement. In contrast, the emission points for H11-H15 are almost constant with the crystal orientation (fig. 3d). Such nearly isotropic response is captured by the phase measurement as well (fig. 3b).

We now turn to resolving the light-induced dressing of the band structure. While in large band gap materials, such as $\mathrm{MgO}$, observing band gap modifications requires high intensities, the gap between the conduction bands is relatively small. Therefore, it can be significantly modified already at moderate field intensities. While these changes are obscured in the single-color high harmonic spectrum, the two-color high harmonic spectroscopy proves to be a sensitive probe of this effect.

We study this phenomenon by focusing on harmonic emission associated with an energy gap between the first and second conduction band. In fig. 4a we plot the field free band structure, which provides a good description of the system at low laser intensities. At $0^{\circ}$ orientation the minimum energy gap between two conduction bands, $\epsilon_{c 2}-\epsilon_{c 1}$, is around $3 \mathrm{eV}$, where harmonic 20 energy is located inside this gap. As we rotate the crystal, the energy gap rapidly reduces to be zero, where harmonic 20 is emitted from the second conduction band. In fig. 4b, we plot the oscillation phase of harmonic 20 as function of the crystal orientation, for different laser intensities. At low intensity (light green plot) the oscillation phase shows a strong angular dependence, mainly between $0^{\circ}-25^{\circ}$. Such an angular 
response captures the dramatic angular variation of the energy gap between the two conduction bands. Importantly, as we increase the field's intensity, the angular dependence of the oscillation phase decreases and then flattens significantly (dark green plots). These experimental results are confirmed by our numerical simulations (fig. 4c). The simulations propagate semiconductor Bloch equations within 7 bands in full dimensionality, using ab-initio band-structure and phaseconsistent coupling matrix elements computed, following the method described in reference ${ }^{[40]}$ (see SI for details). Numerically, the flattening of the angular dependence of the oscillation phase coincides with the onset of strong sub-cycle Landau-Dykhne transitions, with the probability approaching $50 \%$.

Our theoretical and numerical results link these intensity-dependent observations with the closing of the effective band-gap by the laser field. According to equation (2), the oscillation phase is dictated by the additional complex phase, $\sigma$, induced by the $\mathrm{SH}$ field ${ }^{[35]}$. The imaginary component, $\operatorname{Im}(\sigma)$, is associated with perturbations of the tunneling and recombination probabilities, while the real component $\operatorname{Re}(\sigma)$ reflects subtle modifications of the electron trajectory as it propagates within the band. Since the odd and the even harmonics represent constructive and destructive interference of two subcycle emissions, $\operatorname{Re}(\sigma)$ leads to an oscillation phase difference. However the imaginary part, $\operatorname{Im}(\sigma)$, affects both even and odd harmonics simultaneously, therefore their oscillation phases coincide (see SI). The phase difference, $\Phi_{2 N+1}-\Phi_{2 N}$, identifies the balance between the imaginary and the real part of $\sigma$ and the perturbation along the different steps of the interaction.

In fig. 4d, we plot the relative oscillation phase of harmonics 19 and 20, $\Phi_{20}-\Phi_{19}$, at $0^{\circ}$ orientation, for different fundamental field intensities. At sufficient low laser intensity, this phase difference vanishes where the two harmonics oscillate in phase. This response reflects the dominant role of the imaginary component, $\operatorname{Im}(\sigma)$. The origin of this imaginary component could be associated with an anomalous emission event of these harmonics. Indeed, in the free field band structure (fig. 4a), at $0^{\circ}$, harmonics 19 and 20 are emitted from the gap between the two conduction bands. Such emission is accompanied by a large imaginary component in the action that represents the suppressed recombination probability. At higher field's intensities, the band structure is strongly dressed so the energy gap between the bands becomes negligible. In these intensities, the asso- 
ciated imaginary component is reduced, therefore the perturbation is dominated by the real component $\operatorname{Re}(\sigma)$, leading to a phase difference between the even and the odd harmonics. Our experimental results suggest that such a mechanism is reflected by the rapid change in $\Phi_{20}-\Phi_{19}$ with the field's intensity. We note that this observation is unique to harmonics 19 and 20. Outside this spectral range the relative phases associated with emission from the first and second conduction bands (as H17-H18 and H21-H22), show no dependence on the laser intensity (figure 4d). Indeed, these harmonic are not influenced by band gap modifications.

In summary, our study establishes all-optical spectroscopy of a strongly driven crystal, revealing laser-induced modification of the band structure. We identify the dynamical transitions between several conduction bands as well as probe their structural dependence. Importantly, we resolve the clear signature of harmonics emission from an energy gap, between two conduction bands, probing its modification by the laser field. This study provides a general framework for resolving and interpreting attosecond electronic response phenomena in strongly driven solids. Looking forward, two colors HHG spectroscopy opens a window into the observation a broad range of electronic phenomena - from sub-cycle phase transitions to ultrafast dynamics in correlated systems - some of which have been theoretically predicted decades ago, while others are still hotly debated.

\section{References}

[1] Corkum, P. á. \& Krausz, F. Attosecond science. Nature physics 3, 381-387 (2007).

[2] Garg, M. et al. Multi-petahertz electronic metrology. Nature 538, 359 (2016).

[3] Langer, F. et al. Lightwave valleytronics in a monolayer of tungsten diselenide. Nature 557, 76-80 (2018).

[4] Lucchini, M. et al. Attosecond dynamical franz-keldysh effect in polycrystalline diamond. Science 353, 916-919 (2016).

[5] Schultze, M. et al. Attosecond band-gap dynamics in silicon. Science 346, 1348-1352 (2014). 
[6] Schiffrin, A. et al. Optical-field-induced current in dielectrics. Nature 493, 70-74 (2013).

[7] Schultze, M. et al. Controlling dielectrics with the electric field of light. Nature 493, 75 (2013).

[8] Silva, R., Jiménez-Galán, Á., Amorim, B., Smirnova, O. \& Ivanov, M. Topological strong-field physics on sub-laser-cycle timescale. Nature Photonics 13, 849-854 (2019).

[9] Jiménez-Galán, Á., Silva, R., Smirnova, O. \& Ivanov, M. Lightwave control of topological properties in $2 \mathrm{~d}$ materials for sub-cycle and non-resonant valley manipulation. Nature Photonics 14, 728-732 (2020).

[10] Lakhotia, H. et al. Laser picoscopy of valence electrons in solids. Nature $\mathbf{5 8 3}$, 55-59 (2020).

[11] Cohen-Tannoudji, C. N. Nobel lecture: Manipulating atoms with photons. Reviews of Modern Physics 70, 707 (1998).

[12] Haroche, S. Nobel lecture: Controlling photons in a box and exploring the quantum to classical boundary. Reviews of Modern Physics 85, 1083 (2013).

[13] Marangos, J. P. Electromagnetically induced transparency. Journal of Modern Optics 45, 471-503 (1998).

[14] Fleischhauer, M. \& Lukin, M. D. Quantum memory for photons: Dark-state polaritons. Physical Review A 65, 022314 (2002).

[15] Hau, L. V., Harris, S. E., Dutton, Z. \& Behroozi, C. H. Light speed reduction to 17 metres per second in an ultracold atomic gas. Nature 397, 594-598 (1999).

[16] Sokolov, A. V., Walker, D. R., Yavuz, D. D., Yin, G. Y. \& Harris, S. E. Raman generation by phased and antiphased molecular states. Physical review letters 85, $562(2000)$.

[17] Jiangbin, G., Morales-Molina, L., Haenggi, P. et al. Many-body coherent destruction of tunneling. Physical Review Letters 103 (2009). 
[18] Ghimire, S. et al. Observation of high-order harmonic generation in a bulk crystal. Nature physics 7, 138 (2011).

[19] Vampa, G. et al. All-optical reconstruction of crystal band structure. Physical review letters 115, 193603 (2015).

[20] Schubert, O. et al. Sub-cycle control of terahertz high-harmonic generation by dynamical bloch oscillations. Nature Photonics 8, 119 (2014).

[21] Uzan, A. J. et al. Attosecond spectral singularities in solid-state highharmonic generation. Nature Photonics 14, 183-187 (2020).

[22] Tancogne-Dejean, N., Mücke, O. D., Kärtner, F. X. \& Rubio, A. Impact of the electronic band structure in high-harmonic generation spectra of solids. Physical review letters 118, 087403 (2017).

[23] Schiessl, K., Ishikawa, K., Persson, E. \& Burgdörfer, J. Quantum path interference in the wavelength dependence of high-harmonic generation. Physical review letters 99, 253903 (2007).

[24] Wu, M. et al. Orientation dependence of temporal and spectral properties of high-order harmonics in solids. Physical Review A 96, 063412 (2017).

[25] Silva, R., Blinov, I. V., Rubtsov, A. N., Smirnova, O. \& Ivanov, M. Highharmonic spectroscopy of ultrafast many-body dynamics in strongly correlated systems. Nature Photonics 1 (2018).

[26] Jürß, C. \& Bauer, D. Helicity flip of high-order harmonic photons in haldane nanoribbons. Physical Review A 102, 043105 (2020).

[27] Jiménez-Galán, Á., Ivanov, M., Dixit, G. et al. Light-induced valleytronics in pristine graphene. arXiv preprint arXiv:2011.04973 (2020).

[28] Yoshikawa, N., Tamaya, T. \& Tanaka, K. High-harmonic generation in graphene enhanced by elliptically polarized light excitation. Science $\mathbf{3 5 6}$, 736-738 (2017).

[29] Keldysh, L. et al. Ionization in the field of a strong electromagnetic wave. Sov. Phys. JETP 20, 1307-1314 (1965). 
[30] Jürgens, P. et al. Origin of strong-field-induced low-order harmonic generation in amorphous quartz. Nature Physics 16, 1035-1039 (2020).

[31] Vampa, G. et al. Theoretical analysis of high-harmonic generation in solids. Physical review letters 113, 073901 (2014).

[32] Hawkins, P. G. \& Ivanov, M. Y. Role of subcycle transition dynamics in high-order-harmonic generation in periodic structures. Physical Review A 87, 063842 (2013).

[33] You, Y. S. et al. Laser waveform control of extreme ultraviolet high harmonics from solids. Optics letters 42, 1816-1819 (2017).

[34] Dudovich, N. et al. Measuring and controlling the birth of attosecond xuv pulses. Nature physics 2, 781 (2006).

[35] Pedatzur, O. et al. Attosecond tunnelling interferometry. Nature Physics 11, 815 (2015).

[36] Vampa, G. et al. Linking high harmonics from gases and solids. Nature $\mathbf{5 2 2}$, $462(2015)$.

[37] Bruner, B. D. et al. Multidimensional high harmonic spectroscopy of polyatomic molecules: detecting sub-cycle laser-driven hole dynamics upon ionization in strong mid-ir laser fields. Faraday discussions 194, 369-405 (2016).

[38] Vampa, G. et al. Attosecond synchronization of extreme ultraviolet high harmonics from crystals. Journal of Physics B: Atomic, Molecular and Optical Physics 53, 144003 (2020).

[39] You, Y. S., Reis, D. A. \& Ghimire, S. Anisotropic high-harmonic generation in bulk crystals. Nature Physics 13, 345 (2017).

[40] Silva, R., Martín, F. \& Ivanov, M. High harmonic generation in crystals using maximally localized wannier functions. Physical Review B 100, 195201 (2019). 


\section{Methods}

\section{Dressed Bands in Strongly Driven Solids}

We consider the response of a two-band solid driven by a strong low-frequency field, and show how an effective band structure can be introduced in this case on the sub-cycle time-scale, relevant for the low-frequency drivers. In addition to a simple result which averages the instantaneous adiabatic energies over the current wavefunction, we introduce an extension of the Floquet-type analysis to the sub-cycle time-scale.

\section{Floquet-type analysis on the sub-cycle time-scale: Effective band gap} in a strong low frequency field

Let us start with some well defined eigenstate, with the wavefunction $\psi_{\kappa}$, which satisfies the stationary Schroedinger equation, $\hat{H} \psi_{\kappa}=E_{g} \psi_{\kappa}$. Here $\kappa$ collects the quantum numbers that label the state. In a solid, $\kappa$ labels the crystal momentum $\mathbf{k}$ together with the band index $n$.

Let the Hamiltonian depend on some external parameter $\beta, \hat{H}(\beta)$. If we change $\beta$ slowly, our eigenstate will slowly evolve following the stationary Schroedinger equation,

$$
\hat{H}(\beta) \phi_{\kappa}(\beta)=\epsilon_{\kappa}(\beta) \phi_{\kappa}(\beta)
$$

In the low-frequency field, where the driver has frequency $\omega$ substantially lower than the energy gap, such parameter $\beta$ is $\beta=\omega t$. From now on we will simply refer to the time $t$. In the low-frequency field, the adiabatic states are the solutions of the stationary Schroedinger equation with time treated as a parameter:

$$
\begin{aligned}
& \hat{H}(t) \phi_{\kappa}(t)=\epsilon_{\kappa}(t) \phi_{\kappa}(t) \\
& \hat{H}(t)=\hat{H}_{0}+\hat{V}(t)
\end{aligned}
$$

where $V(t)$ is the interaction with the low-frequency field. The adiabatic eigenstates $\phi_{\kappa}(t)$ form complete basis set. Each state has an associated time-dependent state $\Psi_{\kappa}$ which incorporates the standard energy phase-factor:

$$
\Psi_{\kappa}(t)=e^{-i \int_{t_{i}}^{t} \epsilon_{\kappa}\left(t^{\prime}\right) d t^{\prime}} \phi_{\kappa}(t)
$$


Putting this back into the time-dependent Schroedinger equation, we see that the equation for $\Psi_{\kappa}$ contains extra term proportional to the derivative of the eigenstate:

$$
i \dot{\Psi}_{\kappa}=\hat{H}(t) \Psi_{\kappa}+i e^{-i \int_{t_{i}}^{t} \epsilon_{\kappa}\left(t^{\prime}\right) d t^{\prime}} \dot{\phi}_{\kappa}
$$

As long as the non-adiabatic Landau-Dykhne transitions between the adiabatic states, caused by this term, are small, their instantaneous energies $\epsilon_{\kappa}(t)$ offer a very good approximation for the effective instantaneous (and hence sub-cycle) energies of the driven system. However, in the presence of strong non-adiabatic transitions between the adiabatic states, these concepts require corrections.

Consider now the specific case of a solid, with bands $\epsilon_{n}(\mathbf{k})$ and the Bloch wavefunctions $\phi_{n, \mathbf{k}}$, interacting with a low-frequency laser field. We note that the analysis below is not, in fact, limited to such low-frequency case, but the lowfrequency case presents the most natural physical situation where our analysis and its conclusions are physically transparent.

With the light-solid interaction treated in the dipole approximation and in the length gauge, the initial crystal momentum $\mathbf{k}$ becomes a function of time. We shall label this time-dependent momentum $\kappa(t)=\mathbf{k}+\mathbf{A}(t)$.

It is very useful then to use the Houston states to analyze the interaction. In this basis the Bloch wavefunctions $\phi_{n, \mathbf{k}}$ and the band energies $\epsilon_{n}(\mathbf{k})$ also follow the vector potential, replacing $\mathbf{k}$ with $\kappa(t)=\mathbf{k}+\mathbf{A}(t)$. The field-free energy $\epsilon_{n}(\mathbf{k})$ goes into $\epsilon_{n}(\kappa(t)) \equiv \epsilon_{n}(\mathbf{k}+\mathbf{A}(t))$, so that the band energy "slides" with the instantaneous momentum $\kappa(t)$. The Bloch wavefunction also "slides" with this instantaneous momentum, becoming $\phi_{n, \kappa(t)}$.

In this basis, each time-dependent crystal momentum $\kappa(t)=\mathbf{k}+\mathbf{A}(t)$ "traces" its own multi-level system, with its states labeled with the band index $n$ and the time-dependent energies and couplings. The multi-level system with crystal momentum $\mathbf{k}$ is decoupled from other multi-level systems with momenta $\mathbf{k}$ '.

\section{Beyond the adiabatic evolution}

Consider a two-band solid, with the band indexes $n=1,2$, driven by a lowfrequency field. Thanks to the small frequency, the adiabatic evolution would be a good approximation for the most part of the Brillouin zone. The adiabatic evolution will break down to the greatest extent in the regions of the smallest band 
gap, with exponential dependence on the bandgap.

Different $\mathbf{k}$ will reach these regions at different times and hence with different instantaneous values of the field $\mathbf{F}(t)$. Suppose we start in the state $\mathbf{k}$, turn on the field, and at a moment $t_{i}$ arrive at some momentum $\kappa$ in one of the adiabatic states (say with the label $|1, \kappa\rangle$ ), approaching a region where the two bands are coming close to each other.

We shall now look at the propagator across the region of interest. The analysis is general, but for the physical interpretation to be clear, the time-interval should be enough to go through the region. For compactness we will drop the crystal momentum index for the moment.

The propagator, written in the basis of the adiabatic states $\left|\Psi^{(1)}\right\rangle,\left|\Psi^{(2)}\right\rangle$, with energies $\epsilon^{(1)}(t)$ and $\epsilon^{(2)}(t)$, has the following form

$$
\begin{array}{r}
\hat{U}\left(t, t_{i}\right)=e^{-i \frac{1}{2}\left[\lambda^{(1)}+\lambda^{(2)}\right]}\left(\begin{array}{rr}
\cos \alpha e^{i \lambda} & -\sin \alpha e^{i \phi} \\
\sin \alpha e^{-i \phi} & \cos \alpha e^{-i \lambda}
\end{array}\right) \\
\lambda=\frac{1}{2}\left[\lambda^{(2)}-\lambda^{(1)}\right]
\end{array}
$$

This form of the propagator is general and meets the key requirements:

$$
\begin{aligned}
& \left|U_{11}\right|^{2}+\left|U_{21}\right|^{2}=1 \\
& \left|U_{12}\right|^{2}+\left|U_{22}\right|^{2}=1 \\
& \hat{U} \hat{U}^{\dagger}=\hat{U}^{\dagger} \hat{U}=\hat{1}
\end{aligned}
$$

The last point also ensures that the wavefunctions remain orthogonal during the passage, $\left\langle\Psi^{(2)}\left|\hat{U}^{\dagger} \hat{U}\right| \Psi^{(1)}\right\rangle=0$.

The meaning of the matrix elements in this propagator is as follows.

- The phases $\lambda^{(1,2)}(t)$ are associated with the adiabatic energies plus, in general, the geometrical Berry phase:

$$
\lambda^{(i)}(t)=\int_{t_{i}}^{t} d t^{\prime} \epsilon^{(i)}\left(t^{\prime}\right)+\gamma_{i}
$$

The reason we want to complete the passage across the region of interest is that we want the geometrical phase, associated with this passage, to accumulate fully. But, if we treat the problem fully numerically, then, of course, such requirement is not necessary: the phases $\lambda^{(i)}(t)$ are simply found numerically. 
- The overall factor in front of the propagator sets the zero-energy level as

$$
\langle\epsilon\rangle=\frac{1}{2}\left[\epsilon^{(2)}+\epsilon^{(1)}\right]
$$

through the phases associated with the adiabatic energies.

- Irrespective of how the phases $\lambda^{(i)}(t)$ are obtained, their physical interpretation remains the same: their time-derivatives have to be associated with the adiabatic energies (which are viable and meaningful in the absence of non-adiabatic transitions, i.e. when $|\sin \alpha|<<1)$ :

$$
\tilde{\epsilon}^{(i)}(t)=\frac{\partial \lambda^{(i)}(t)}{\partial t}
$$

The reason to add "tilde" above the adiabatic energy is to stress that the adiabatic energies $\tilde{\epsilon}^{(i)}(t)$ obtained in such way may not always coincide with the adiabatic energies obtained by diagonalizing the adiabatic Hamiltonian, because of the presence of the geometric phase. Again, we stress that the phases associated with $\tilde{\epsilon}^{(i)}(t)$ can be extracted numerically. For the physical interpretation we will need are their derivatives.

- The off-diagonal elements describe the Landau-Zener-Dykhne non-adiabatic transitions between the two adiabatic states.

- The phases $\phi$ of these off-diagonal elements are determined by the landscape of the bands and the Berry connections (couplings). As we shall see below, $\phi$ will not matter for the effective band structure.

- The probability of staying in the adiabatic state is $\cos ^{2} \alpha$ and the probability of making the transition is $\sin ^{2} \alpha$.

We can now develop the sub-cycle version of the Floquet analysis. To this end, we return to the propagator, dropping the common zero-energy level phase factor for compactness,

$$
\begin{array}{r}
\hat{U}\left(t, t_{i}\right)=\left(\begin{array}{rr}
\cos \alpha e^{i \lambda} & -\sin \alpha e^{i \phi} \\
\sin \alpha e^{-i \phi} & \cos \alpha e^{-i \lambda}
\end{array}\right) \\
\lambda=\frac{1}{2}\left[\lambda^{(2)}-\lambda^{1)}\right]
\end{array}
$$


and look for orthogonal states $\left|\Psi^{\mu}\left(t_{i}\right)\right\rangle$ with time-dependent quasi-energies $\epsilon^{\mu}\left(t, t_{i}\right)$, which depend on the crystal momentum $\mathbf{k}$. We want these states to behave as if they were the Floquet states for this propagator:

$$
\begin{array}{r}
\hat{U}\left(t, t_{i}\right)\left|\Psi^{\mu}\right\rangle=e^{i \mu}\left|\Psi^{\mu}\right\rangle \\
\epsilon^{\mu}=-\frac{\partial \mu}{\partial t}
\end{array}
$$

The positive sign in the phase of the exponent is for convenience because we shall start with the "lower" state, which has negative energy. The minus sign in the equation for the quasi-energy is related to the positive sign in the exponent in the first equation.

These quasi-energies and the associated states are as close as one can get to the effective bands and effective eigenstates in a strongly driven system with nonadiabatic transitions. As we shall see below, in the absence of non-adiabatic transitions they, of course, coincide with the adiabatic energies and states.

In principle, one can try to find such states for any time-interval after $t_{i}$, but a meaningful time-interval is an interval sufficient to cross the transition region. Once the region is crossed, each component of the wavefunction, projected on the adiabatic states, will mostly evolve on the associated adiabatic bands, and in the absence of non-adiabatic transitions these bands are fine and, as mentioned above, coincide with the quasi-energies we shall find below.

The time-dependent "eigenstates" of the propagator have two components corresponding to the amplitudes in the two adiabatic states $\left|\phi^{(i)}(t)\right\rangle$,

$$
\left|\Psi^{\mu}\right\rangle=\left(\begin{array}{c}
a_{\mu} \\
b_{\mu}
\end{array}\right)
$$

The analysis is straightforward. We ask that the determinant of the matrix $\hat{U}-e^{i \mu} \hat{1}$ is equal to zero, and the solution is found to be

$$
\cos \mu=\cos \alpha \cos \lambda
$$

There are two solutions of this equation, $\mu_{1}=\mu$ and $\mu_{2}=-\mu$, and the quasienergies $\epsilon^{\mu_{1}}, \epsilon^{\mu_{2}}$ are obtained by differentiating $\mu_{1}$ and $\mu_{2}$ with respect to time.

The first observation is that, in the absence of non-adiabatic transitions, when $\cos \alpha=1, \cos \mu=\cos \lambda, \mu_{1}=-\lambda$ and $\mu_{2}=\lambda$, and the quasi-energies coincide with the adiabatic states. 
Eq.(15) is already sufficient to find the effective bandgap, which is equal to

$$
\Delta \epsilon^{\mu}=\left|\epsilon^{\mu_{1}}-\epsilon^{\mu_{2}}\right|=2\left|\frac{\partial \mu}{\partial t}\right|
$$

We differentiate the two sides of Eq.(15) with respect to time and find

$$
\Delta \epsilon^{\mu}=\left[\tilde{\epsilon}_{\mathrm{ad}}^{(2)}-\tilde{\epsilon}_{\mathrm{ad}}^{(1)}\right]\left|\cos \alpha \frac{\sin \lambda}{\sin \mu}\right|
$$

where we have used that

$$
2 \dot{\lambda}=\tilde{\epsilon}_{\mathrm{ad}}^{(2)}-\tilde{\epsilon}_{\mathrm{ad}}^{(1)}
$$

Using the relationship $\cos \mu=\cos \alpha \cos \lambda$, we can re-write

$$
\left[\cos \alpha \frac{\sin \lambda}{\sin \mu}\right]^{2}=\frac{\cos ^{2} \alpha \sin ^{2} \lambda}{\cos ^{2} \alpha \sin ^{2} \lambda+\sin ^{2} \alpha}
$$

and hence

$$
\Delta \epsilon^{\mu}=\left[\tilde{\epsilon}_{\mathrm{ad}}^{(2)}-\tilde{\epsilon}_{\mathrm{ad}}^{(1)}\right] \sqrt{\frac{\cos ^{2} \alpha \sin ^{2} \lambda}{\cos ^{2} \alpha \sin ^{2} \lambda+\sin ^{2} \alpha}}
$$

Finally, we can simplify this expression by taking into account that if the action $\lambda(t)$ is large, which is usually the case in the strong, low-frequency field, then $\sin ^{2} \lambda\left(t, t_{i}\right)$ is a fast-oscillating function of $t-t_{i}$. Replacing it with its average, $\sin ^{2} \lambda\left(t, t_{i}\right)=>1 / 2$, and introducing the notation $\sin ^{2} \alpha=w_{\mathrm{LD}}$, we get the final result for the bandgap between the two quasi-energies

$$
\Delta \epsilon^{\mu}=\left[\tilde{\epsilon}_{\mathrm{ad}}^{(2)}-\tilde{\epsilon}_{\mathrm{ad}}^{(1)}\right] \sqrt{\frac{1-w_{\mathrm{LD}}}{1+w_{\mathrm{LD}}}}
$$

This result shows that the band gap collapses when the non-adiabatic LandauDykhne transition approaches unity, $w_{\mathrm{LD}} \simeq 1$.

We note that the formalism based on the sub-cycle analogue of the quasi-energy states of a strongly driven system is especially attractive because it naturally merges into the Floquet analysis when $t-t_{i}$ is equal to one period.

One can also find the quasi-eigenergies in a different way, by using only the relationship $\cos \mu=\cos \alpha \cos \lambda$. Namely, one can use this relationship to solve the equations for the amplitudes $a^{\mu}$ and $b^{\mu}$, and then average the full Hamiltonian over the eigenvectors obtained in such way from Eq.(14). The result is exactly the same, as expected. 


\section{Data availability}

The data that support the plots within this paper and other findings of this study are available from the corresponding author upon reasonable request.

\section{Acknowledgements}

N.D. is the incumbent of the Robin Chemers Neustein Professorial Chair. N.D. acknowledges the Minerva Foundation, the Israeli Science Foundation and the European Research Council for financial support. A.J.N.U. acknowledges financial support by the Rothschild Foundation and the Zuckerman Foundation. M.I. acknowledges funding of the DFG QUTIF grant IV152/6-2. A.J.G. and M.I. acknowledge funding from the European Union's Horizon 2020 research and innovation program under grant agreement No 899794.

\section{Contributions}

N.D. and M.I. supervised the study. M.I. supervised the theoretical work and developed the theoretical model. A.J.N.U. and G.O. conceived and planned the experiments. A.J.G. and R.S. performed the theoretical study and the numerical analysis. A.J.N.U., T.A.P., G.O. and B.D.B performed the measurements. A.J.N.U., G.O. and S.S. analyzed the data. B.Y. performed the DFT calculations. All authors discussed the results and contributed to writing the manuscript.

\section{Competing financial interests}

The authors declare no competing financial interests.

\section{Corresponding authors}

Correspondence to Nirit Dudovich (nirit.dudovich@weizmann.ac.il). 


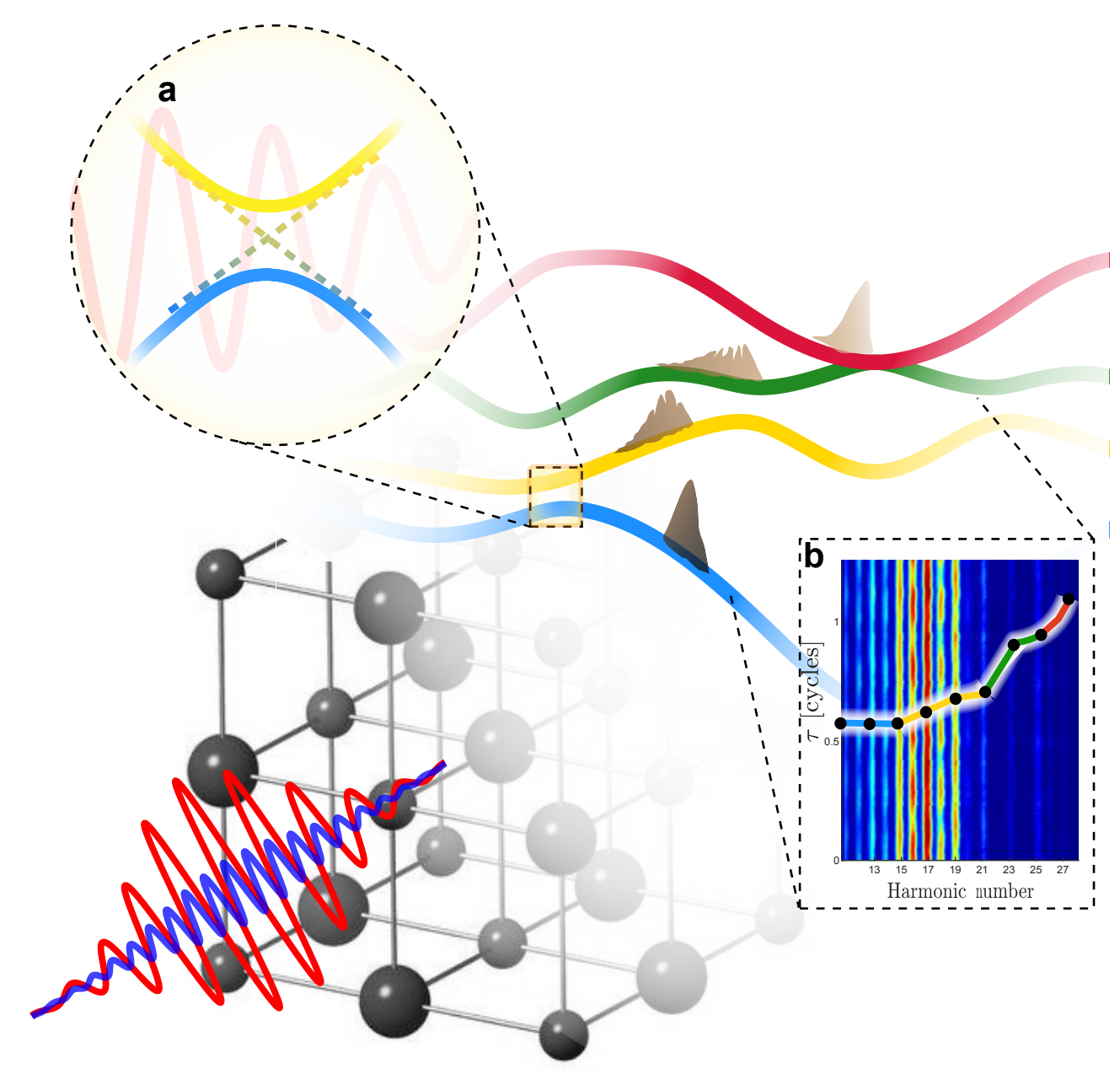

Figure 1: All optical spectroscopy of dynamical band structure. a, Nonadiabatic Landau-Dykhne transition between a pair of bands. b, Two color HHG spectroscopy probes the internal dynamics, mapping the temporal properties of electron trajectories, transitions between the bands as well as their laser driven modifications. 

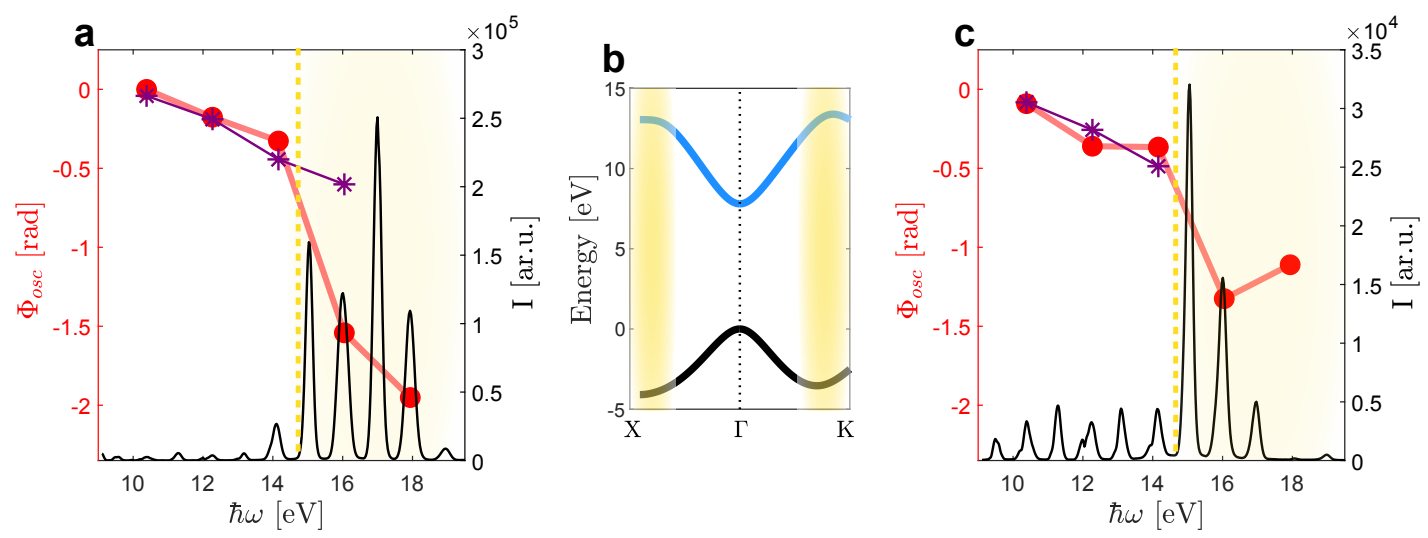

Figure 2: HHG spectroscopy beyond the semi-classical description a,c HHG spectrum (black line) and the oscillation phase (red dots) as function of photon energy for crystals orientations of $0^{\circ}(\mathrm{a})$ and $45^{\circ}(\mathrm{c})$. Calculated oscillation phase using the saddle point approximation in interband model ${ }^{[31]}$ (purple star markers). b, The valence and the first conduction band for $0^{\circ}(\Gamma$ to $X)$ and for $45^{\circ}$ $(\Gamma$ to $K)$. The yellow shaded area emphasizes the energy range where the semiclassical description fails ${ }^{[21]}$, also marked at the corresponding photon energies in a and c (dashed yellow line). 

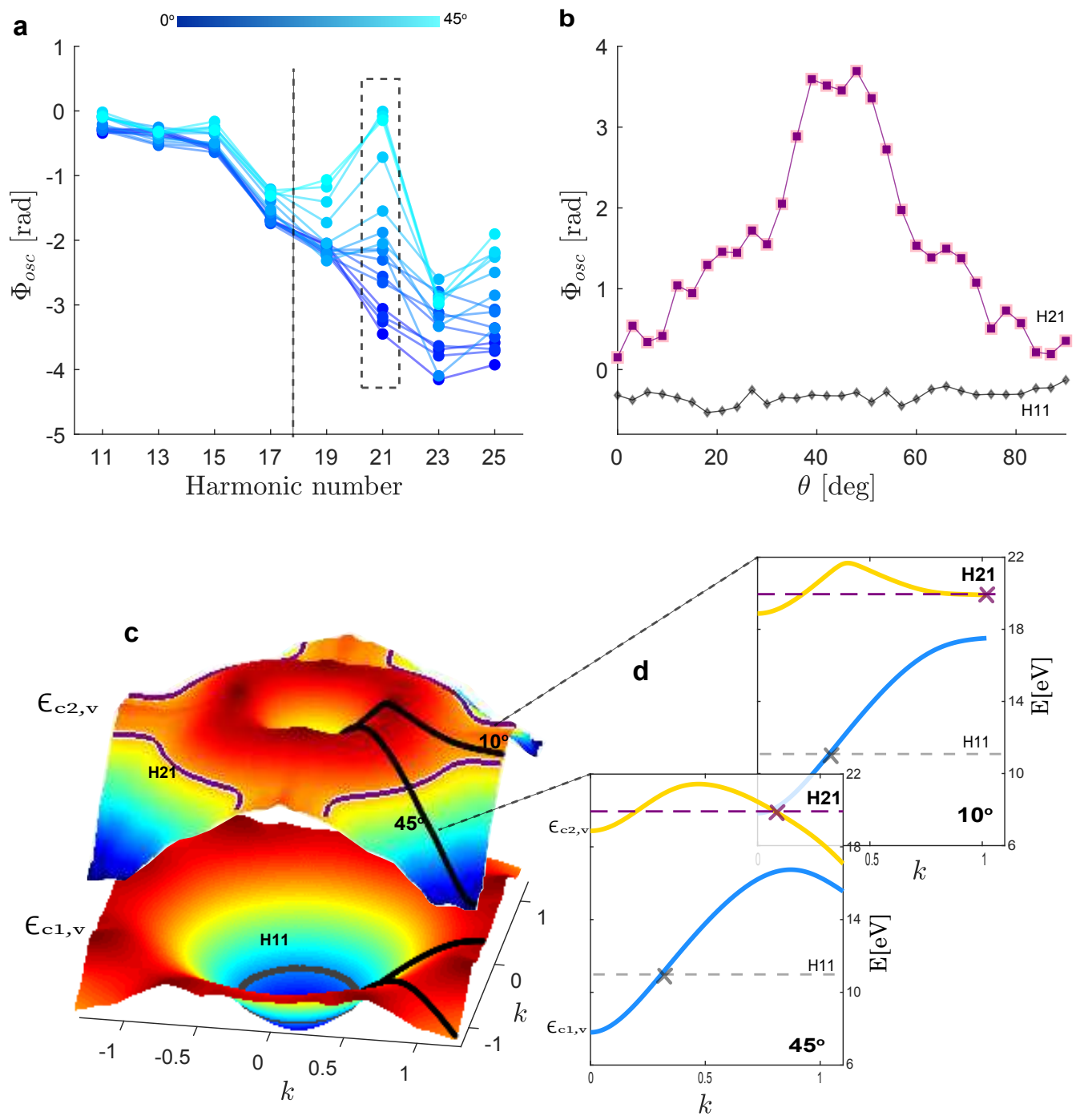

Figure 3: Probing the structural dependence of multiple conduction bands a, The oscillation phase as a function of harmonic number, for different crystal orientation, ranging from $0^{\circ}$ (dark blue) to $45^{\circ}$ (cyan). The dash line marks the cutoff of the first conduction band and the dash box emphasizes H21 oscillation phases for different orientation, plotted at b. b, Harmonic 21 (purple) and harmonic 11 (gray) oscillation phase as a function of crystal orientation. c, top: 2D second band gap, $\epsilon_{c 2, v}=\epsilon_{c 2}-\epsilon_{v}$, as function of crystal momentum. The purple contour represents harmonic 21 energy along different crystal's orientation. bottom: 2D first band gap, $\epsilon_{c 1, v}=\epsilon_{c 1, v}-\epsilon_{v}$, as function of crystal momentum. The gray contour represents harmonic 11 enfergy along different crystal's orientation. d, $1 \mathrm{D}$ cut of the second (yellow) and first (blue) band gap along $10^{\circ}$ and $45^{\circ}$ crystal orientation. The energy of Harmonic 21 and 11 is presented by the purple and gray dash lines as well as their crossing point with the band gaps (cross markers). 

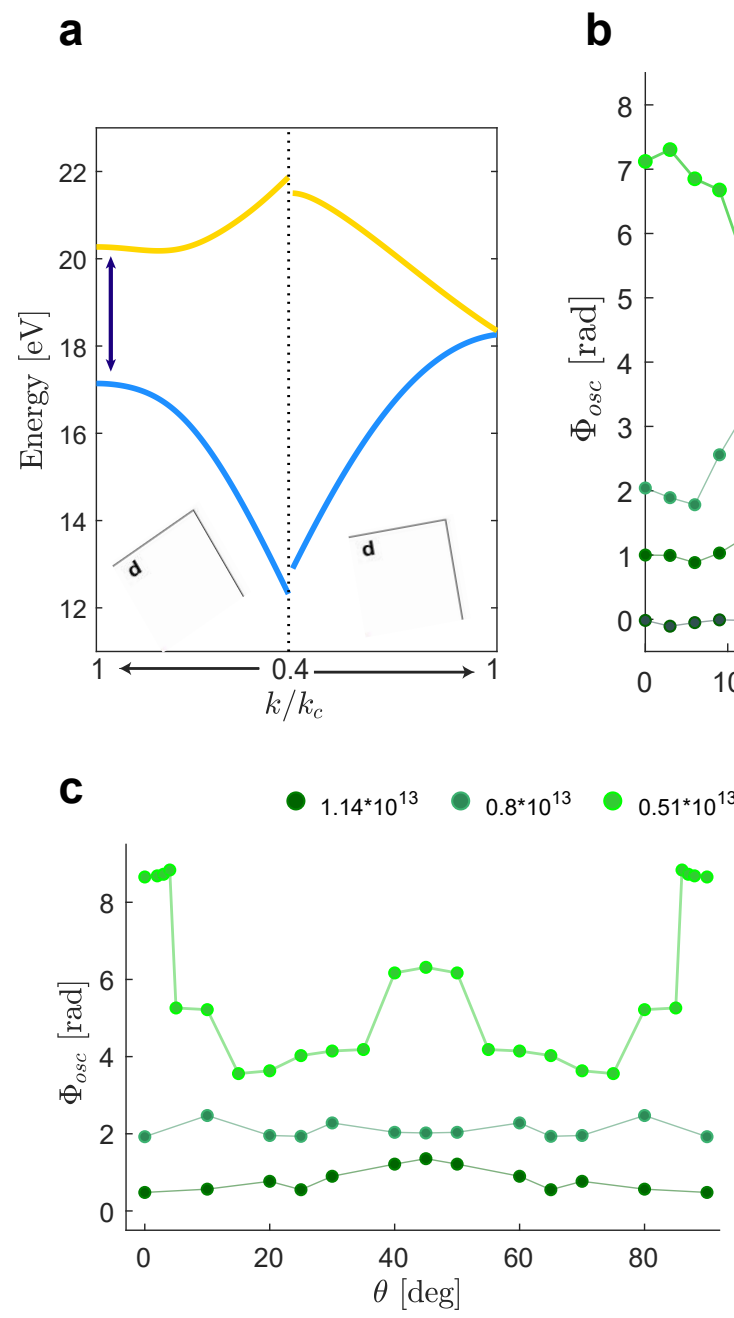

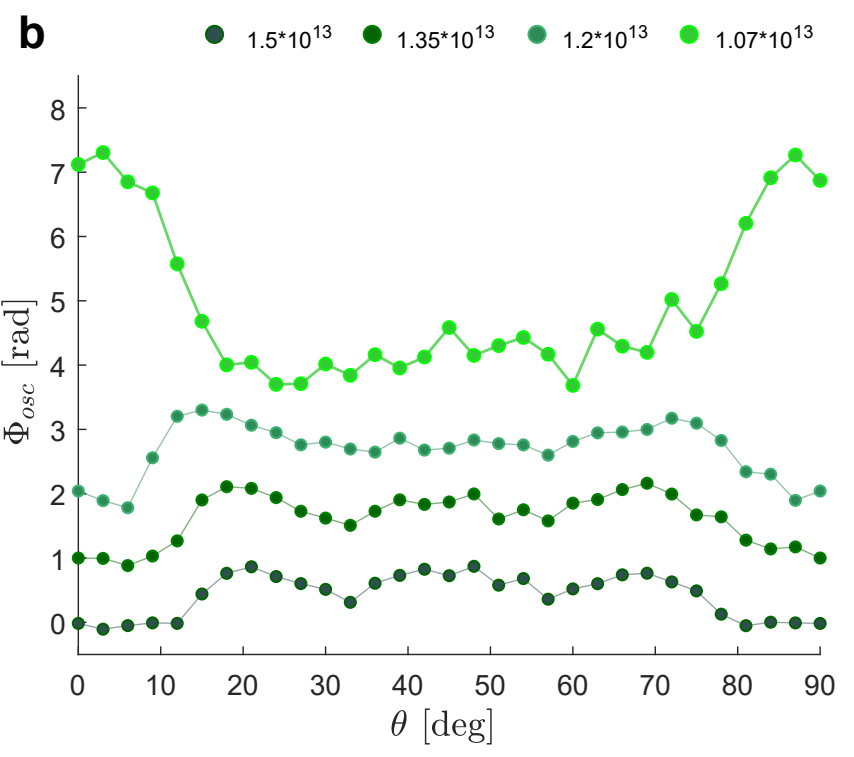

d

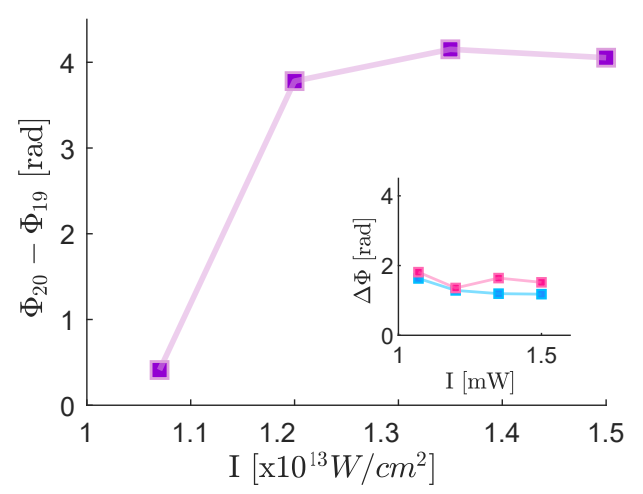

Figure 4: Dynamical band structure. a, First (blue) and second (yellow) band gaps, $\epsilon_{c 1, v}$ and $\epsilon_{c 2, v}$, for $0^{\circ}$ (right) and $25^{\circ}$ (left) orientations, as a function of $k / k_{c}\left(k_{c}=2 \pi / a\right.$, where $a$ the lattice constant). b. Harmonic 20 oscillation phase as function of crystal orientation, for different fundamental field intensities (light green to dark green). c, Calculated oscillation phase of H20 as function of the crystal's orientation, for different fundamental field intensities (light green to dark green) d, The oscillation phase difference,$\Phi_{20}-\Phi_{19}$, at $0^{\circ}$ orientation as function of the fundamental field intensities. Inset: $\Phi_{18}-\Phi_{17}$ (pink) plot and $\Phi_{22}-\Phi_{21}$ (blue). 


\section{Supplementary Files}

This is a list of supplementary files associated with this preprint. Click to download.

- SI.pdf 